\title{
Bacteremia in Critically III Patients with SARS-CoV 2 Infection
}

\author{
Cabello-Modesto Daniel ${ }^{1,5}$, Garcia-Briones Alondra ${ }^{2,5}$, Gutierrez-Villaseñor Alan Omar ${ }^{2,5}$, \\ Aguilar-Zapata Daniel $^{3,5}$, Romero-Gonzalez Juan Pablo ${ }^{2,5}$, Gonzalez-Chon Octavio ${ }^{2,5}$, \\ Juárez-Hernández Eva ${ }^{4,5}$, Santillan-López Gloria ${ }^{3}$, Sosa-Garcia Jesus Ojino ${ }^{2,5, \text { * }}$
}

${ }^{1}$ Internal Medicine Department, Medica Sur Hospital, Mexico City, Mexico

${ }^{2}$ Intensive Care Unit, Medica Sur Hospital, Mexico City, Mexico

${ }^{3}$ Hospital Epidemiological Surveillance Unit, Medica Sur Hospital, Mexico City, Mexico

${ }^{4}$ Translational Research Unit, Medica Sur Hospital, Mexico City, Mexico

${ }^{5}$ Medicine Faculty, Mexico's Autonomous National University, Mexico City, Mexico

Email address:

drintervista@gmail.com (Sosa-García J. O.)

${ }^{*}$ Corresponding author

\section{To cite this article:}

Cabello-Modesto Daniel, Garcia-Briones Alondra, Gutierrez-Villaseñor Alan Omar, Aguilar-Zapata Daniel, Romero-Gonzalez Juan Pablo, Gonzalez-Chon Octavio, Juárez-Hernández Eva, Santillan-López Gloria, Sosa-Garcia Jesus Ojino. Bacteremia in Critically Ill Patients with SARS-CoV 2 Infection. Clinical Medicine Research. Vol. 10, No. 4, 2021, pp. 116-120. doi: 10.11648/j.cmr.20211004.12

Received: June 19, 2021; Accepted: July 1, 2021; Published: July 6, 2021

\begin{abstract}
Background: Since December 2019, the COVID-19 pandemic has caused the dead of 1 million people, from these critically ill patients have an increased risk of bacteremia. Material and Methods: This observational, retrospective, single-center study included 129 critically ill COVID-19 patients with a bacteremia. We studied the clinical characteristics, comorbidities, hospital and intensive care unit length of stay, days on invasive mechanical ventilation, maximum dose of norepinephrine and mortality. Results: From 129 patients were reported 17 patients (13.2\%) with bacteremia. 35.3\% were cataloged as a primary bacteremia. The source of secondary bacteremia was a ventilator associated pneumonia in $81.8 \%$, central line-associated blood stream infection in $18.1 \%$ and urinary catheter infection in $9 \%$. The patients with bacteremia, had a hospitalization stay of 23 days Vs. 20.5 days in the patients without bacteremia ( $p=0.19) ; 18$ Vs. 13.5 days in the ICU ( $=0.061$ ); 15 Vs 11 days on IMV $(p=0.053)$ and a maximum dose of norepinephrine of $0.28 \mathrm{Vs} .0 .11 \mathrm{mcg} / \mathrm{kg} / \mathrm{min}(\mathrm{p}=0.02)$. We reported a $14.8 \%$ of mortality in patients with bacteremia vs. $12.7 \%$ in patients without bacteremia, odds ratio of 0.87 ( $p=0.75$ ). Conclusion: Critically ill COVID-19 patients and bacteremia tend to increase the length of stay in the ICU and days on IMV with no change in mortality.
\end{abstract}

Keywords: COVID-19, SARS-CoV-2, Bacteremia, Critically III Patients

\section{Introduction}

Since December 2019, the COVID-19 pandemic has affected more than 42 million people and caused the death of approximately 164 million people. Se In Mexico there are 891 160 cumulative cases and 88924 deaths until October 2020, according to the World Health Organization (WHO) [1].

It is known that the primary risk factors for clinical worsening and mortality in COVID-19 patients are advanced age and comorbidities, including obesity, hypertension, and diabetes [2]. In Mexican population people among 20 years have an overweight and an obesity prevalence of $72 \%$ with an increment in the period from 2000 to 2016 of $41.8 \%$ in overweight and a $290.5 \%$ in obesity prevalence [3].

There is an increased number of blood stream infections (BSI) in COVID-19 hospitalized patients in the intensive care unit (ICU) after their need of an invasive device like a central venous catheter, an extracorporeal membrane oxygenation (ECMO) or renal replacement therapy $[4,5]$. But there are still few studies aiming on the detection and clinical relevance of bacteremias in COVID-19 patients.

The aim of this study is to describe the clinical and microbiological characteristics of critically III COVID-19 patients with a positive blood culture, identifying the source 
of the bacteremia and the association of the outcome of these patients.

\section{Objective}

The objective of this study was to compare the clinical characteristics of critically III COVID-19 patients and a positive blood culture with patients with negative blood cultures, determining the source of the bacteremia, pathogen, and clinical outcomes, like hospital and ICU days of stay, days on IMV, maximum dose of vasopressor and hospital mortality.

\section{Definitions}

COVID-19 was defined as a patient with a positive RT-PCR test for SARS-CoV-2 on at least one respiratory specimen (either from a nasopharyngeal swab or a BAL). Primary bacteremia was cataloged as at least one positive blood culture with no confirmed source, assuming direct inoculation. Secondary bacteremia was cataloged as at least one positive blood culture with a confirmed source or other positive cultures in specific sites (respiratory or urinary cultures). These definitions and the categorization of the patient bacteremias were made by the HESU. Polymicrobial infections were considered as separate events, one for each causative organism isolated from the blood culture.

\section{Material and Methods}

We conducted a retrospective analysis on patients with COVID-19 admitted to the ICU of Medica Sur Hospital in Mexico City, from March to August 2020. The protocol was authorized by the Research and Ethics Committee of the hospital (2020-EXT-461). We collected the patient's data: gender, age, body mass index (BMI), comorbidities (including smoking, hypertension, heart arrhythmias, diabetes, ischemic cardiac disease, dyslipidemia, chronic pneumopathies, solid and hematologic neoplasm) from the digital medical records. We collected the positive blood culture during the first 14 days of stay in the ICU describing the causal microorganism and determining the source of the bacteremia concomitant with respiratory and urinary cultures. The final determination between a primary or secondary bacteremia was realized by the Hospital Epidemiological Surveillance Unit (HESU). Once determining the source of the bacteremia, we divided in two groups (patients with a bacteremia and patients without a bacteremia) for the comparison of clinical outcomes.

\subsection{Inclusion Criteria}

We included men and women of 18 years and older, with COVID-19 confirmed with a RT-PCR from a nasopharyngeal swab or bronchoalveolar lavage (BAL), requiring invasive mechanical ventilation (IMV), whit at least a 7 day in hospital stay.

\subsection{Exclusion Criteria}

There were excluded patients with blood cultures with isolation of Coagulase negative Staphylococcus, considered as a contaminant, we also excluded pregnant patients.

\subsection{Statistical Analysis}

All data analysis was anonymized and collected, the categorical data was reported in percentages and proportions, continuous data was expressed in medians and interquartile ranges (IQR). The Statistical analysis and associations were realized using Statistical Package for the Social Sciences (SPSS) software version 22.0, the quantitative data was analyzed with a Mann-Whitney $U$ test and qualitative data was analyzed with a chi-square test. The graphics were realized on GraphPad Prism 5.07. The statistical significance on results was stablished with a p value below 0.05 .

\section{Results}

Table 1. Patient's characteristics and comorbidities.

\begin{tabular}{llll}
\hline Characteristics & Total $(\mathbf{n}=\mathbf{1 2 9})$ & Without bacteremia $(\mathbf{n = 1 1 2})$ & With bacteremia $(\mathbf{n = 1 7 )}$ \\
\hline $\begin{array}{l}\text { Age }(\text { years), median [interquartile range] } \\
\text { Gender }\end{array}$ & $59[48-68]$ & $58.5[48-67]$ & \\
Male & $109(84.5 \%)$ & $95(84.8 \%)$ & $143.5-74.5]$ \\
Female & $20(15.5 \%)$ & $17(15.2 \%)$ & $14(82.4 \%)$ \\
BMI $\left(\mathrm{kg} / \mathrm{m}^{2}\right)$, media [interquartile range] & $29.7[26.5-32.9]$ & $29.7[26.6-32.5]$ & $3(17.6 \%)$ \\
Comorbidities & & & $29.4[25.2-33.4]$ \\
Smokers & $8(6.2 \%)$ & $6(5.3 \%)$ & $2(11.7 \%)$ \\
Hypertension & $42(32.5 \%)$ & $36(32.1 \%)$ & $6(35.2 \%)$ \\
Cardiac arrhythmia & $8(6.2 \%)$ & $7(6.2 \%)$ & $1(5.8 \%)$ \\
Diabetes & $36(27.9 \%)$ & $29(25.8 \%)$ & $7(41.1 \%)$ \\
Prior acute ischemic cardiopathy & $6(4.6 \%)$ & $5(4.4 \%)$ & $1(5.8 \%)$ \\
Peripheric arterial disease & $3(2.3 \%)$ & $3(2.6 \%)$ & $0(0 \%)$ \\
Dyslipidaemias & $9(6.9 \%)$ & $8(7.1 \%)$ & $1(5.8 \%)$ \\
Chronic pneumopathy & $3(2.3 \%)$ & $3(2.6 \%)$ & $0(0 \%)$ \\
Ambulatory oxygen user & $1(0.7 \%)$ & $1(0.8 \%)$ & $0(0 \%)$ \\
Solid neoplasm & $9(6.9 \%)$ & $8(7.1 \%)$ & $1(5.8)$ \\
Hematologic neoplasm & $1(0.7 \%)$ & $1(0.8 \%)$ & $0(0 \%)$ \\
\hline
\end{tabular}


A total of 129 patients with confirmed SARS-CoV-2 were identified during the study period (March 1st to August 15th, 2020). The median of age was 57.9 years old [IQR $48-68$ ], with $84.5 \%(n=109)$ male patients and $15.5 \%(n=20)$ female patients. The median of BMI was $30.23 \mathrm{~kg} / \mathrm{m}^{2}$ [IQR 26.5-32.9]. 32.5\% $(n=42)$ of the patients had hypertension, $27.9 \%(\mathrm{n}=36)$ had diabetes, 6.2\% $(\mathrm{n}=8)$ had a cardiac arrhythmia, $6.2 \%(n=8)$ were smokers, and $4.65 \%(n=6)$ had a prior acute ischemic cardiopathy (Table 1).

From all the 129 patients, $13.2 \%$ of patients $(n=17)$ had a positive blood cultures; from those, 17 patients $35.3 \%(n=6)$ were cataloged as a primary bacteremia where Chryseobacterium indologenes was the most common pathogen (2 patients). E. coli, Streptococcus oralis, Streptococcus constellatus, and Streptococcus mitis represented one patient each. Secondary bacteremia was reported in $64.7 \%(n=11)$ of the patients; where the most common pathogens where Pseudomonas aeruginosa and Enterococcus faecalis (4 patients), Staphylococcus aureus (3 patients), Stenotrophomonas maltophilia, Enterococcus faecium, Escherichia coli, and Klebsiella pneumoniae (1 patient each).

In four patients two pathogens were isolated, two had a Gram-negative bacteremia and the other two had mixed microorganism bacteremia (Gram positive and Gram-negative rods) (Figure 1).

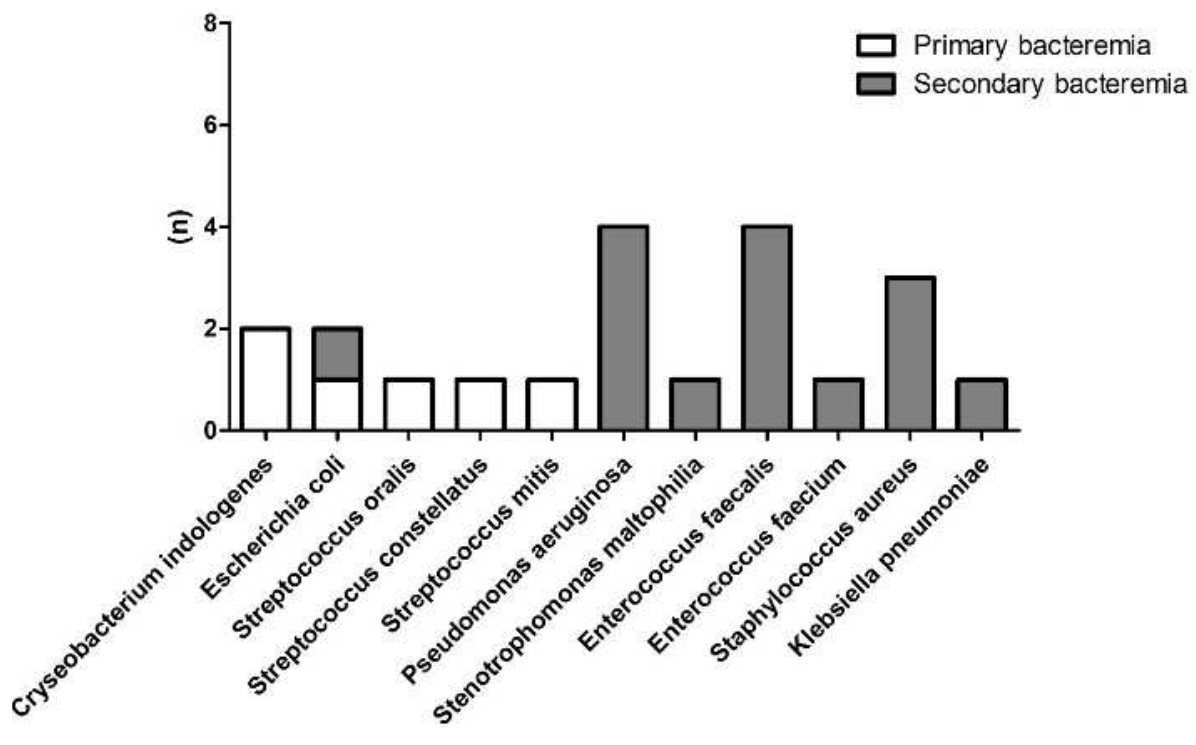

Figure 1. Isolated microorganisms in blood cultures.

The source of secondary bacteremia was a ventilator associated pneumonia (VAP) in $81.8 \%(n=9)$ with isolation of Enterococcus faecalis $(n=3)$, Staphylococcus aureus $(n=1)$, Pseudomonas aeruginosa $(n=4)$ and Klebsiella pneumoniae $(\mathrm{n}=1)$; Central line-associated blood stream infection (CLABSI) in $18.1 \%(n=2)$ with isolation of Staphylococcus aureus $(n=2)$; and urinary catheter infection (UCI) in $9 \%(n=1)$ with isolation of Enterococcus faecalis ( $\mathrm{n}=1)$ (Figure 2).

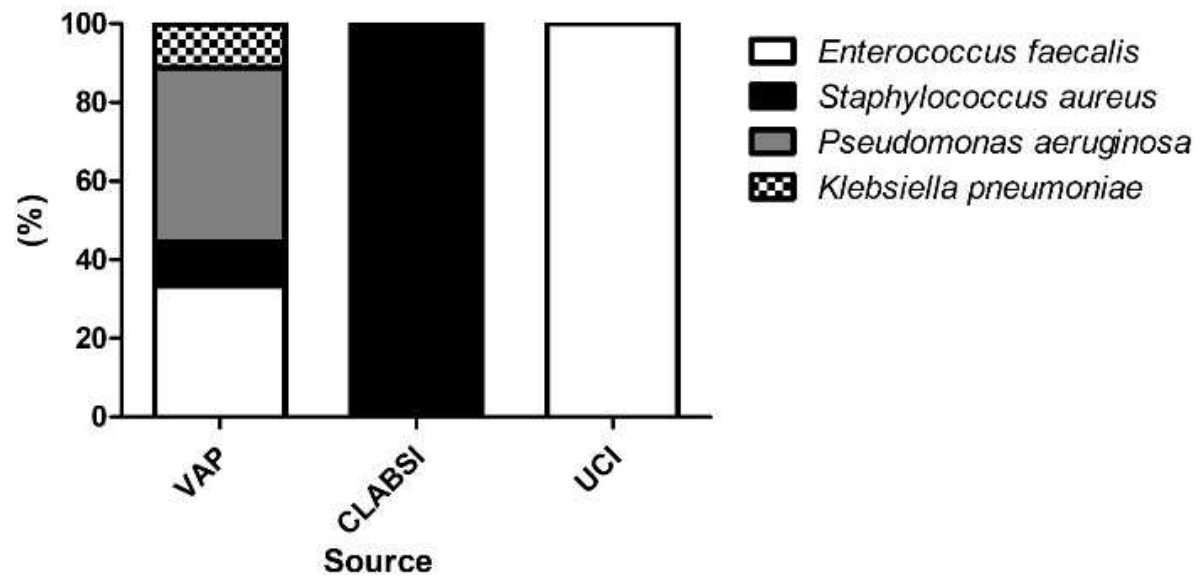

Figure 2. Secondary bacteremias source of infection and pathogens.

Overall, we found a median of 21 [IQR 15.5 - 31.5] days of hospitalization stay, 14 [IQR 9 - 21] days of ICU stay, with a median of 11 [IQR 6 - 19.5] days on IMV and a median of
0.14 [IQR $0.05-0.40] \mathrm{mcg} / \mathrm{kg} / \mathrm{min}$ dose of norepinephrine. The patients with a positive blood culture, a median of total hospitalization stay was 23 days [IQR 15 - 31.7] compared to 
20.5 days [IQR 20 - 31.5] in the patients with negative blood cultures $(p=0.19)$. A median of 18 days [IQR $11-23.5$ ] in the ICU was found in the group of positive blood cultures compared to 13.5 days [IQR 8 - 21] in the group of negative cultures. $(p=0.061)$. The patients with a positive blood cultured had more days under IMV compared to the group of patients with negative blood cultures 15 days [IQR 8.5-19.5] vs 11 days [IQR 6-19.5] respectively $(\mathrm{p}=0.053)$. A median of maximum dose of norepinephrine were $0.28 \mathrm{mcg} / \mathrm{kg} / \mathrm{min}$

a)

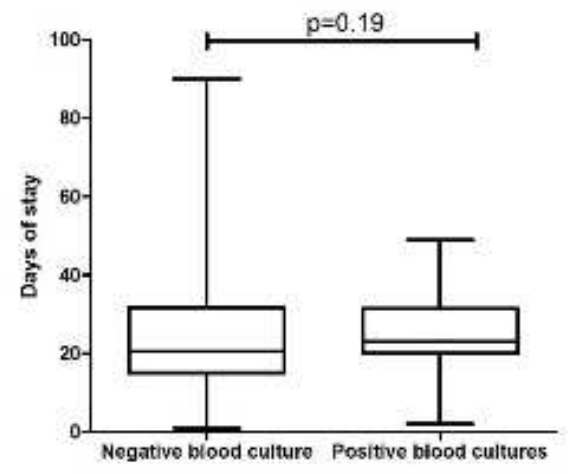

c)

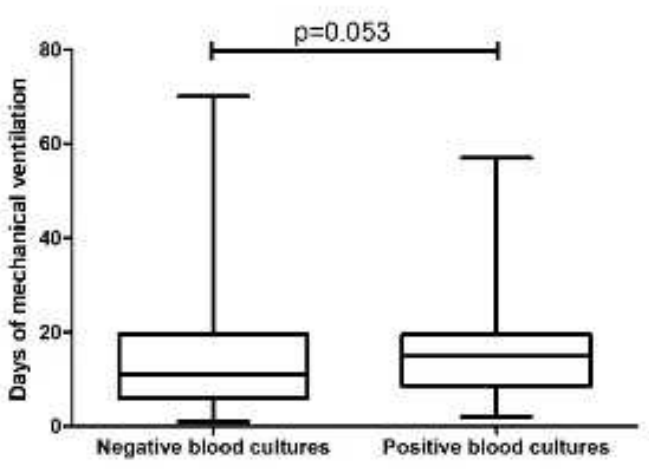

[IQR $0.15-0.5]$ in the positive blood culture group vs. 0.11 $\mathrm{mcg} / \mathrm{kg} / \mathrm{min}$ [IQR $0.05-0.4$ ] in the negative blood culture group ( $\mathrm{p}=0.02$ ) (Figure 3$)$.

The mortality was reported in $13.2 \%(n=27)$ of all the patients; $14.8 \%(n=4)$ for the patients with positive blood culture and $12.7 \%(n=23)$ for the patients with negative blood cultures with an odds ratio (OR) for mortality in patients with a positive blood culture of 0.87 [IQR $0.344-2.21$ ] $(\mathrm{p}=0.75)$ (Figure 3).

b)

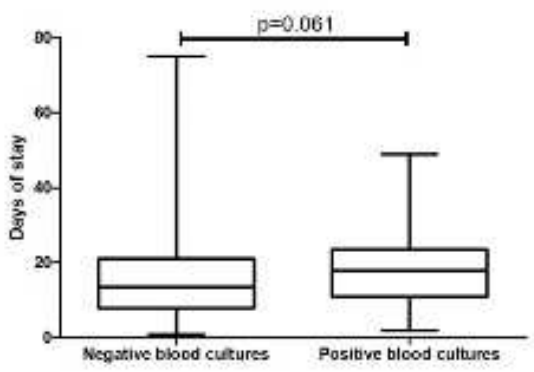

d)

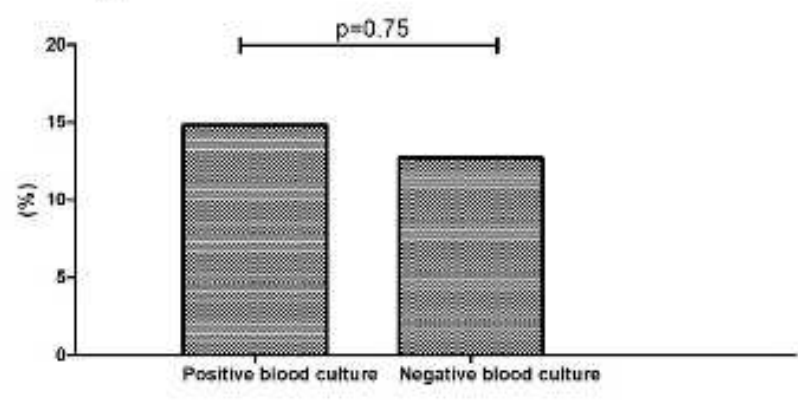

Figure 3. Clinical outcomes in critically III COVID-19 patients with bacteremia. a) Median of hospital days of stay of patients with and without a bacteremia; b) Median of ICU days of stay of patients with and without a bacteremia; c) Median of days on IMV in patients with and without a bacteremia; d) Mortality of patients with and without a bacteremia.

\section{Discussion}

We describe the clinical characteristics of COVID-19 patients hospitalized in ICU with or without a bacteremia and their clinical relevance. In the demographic data we can observe the presence of a middle age population with an elevated BMI and almost one third of them with hypertension and diabetes. The primary strength of this study is the population evaluated because there are no studies in Hispanic population with these comorbidities and in critically III COVID-19 patients that describe the pathogens and characteristics of bacteremia making an association with the hospital and ICU length of stay, days on IMV and mortality.

The presence of a positive blood culture was reported in $13.2 \%(n=17)$ of the patients, greater than other studies, like in Engsbro, et al., were the presence of blood stream infections was demonstrated in $5.3 \%$ of their critically III patients $(n=12)$ [6]; in Yu and cols that reported the presence of a positive blood culture in $6.5 \%$ of their patients; and Martinez-Guerra and cols. [7] they observed the presence of a blood stream infections on $29.1 \%$ of their population $(n=32)$ being a coagulase negative staphylococci the most frequent cause in $40 \%$, followed by Enterobacter complex in $20 \%$ with a $14.3 \%$ of AmpC producers pathogens, $2.9 \%$ Extended spectrum beta lactamases and $2.9 \%$ Pseudomonas aeruginosa multidrug resistant, nevertheless this last studies does not make difference between mild, moderate or critically III patients $[7,8]$.

Bacteremia related to other sources was described by Sligl and colleagues where the most common source of bacteremia was an E. coli associated pneumonia in 33\% [9]. However, the studies from Engsbro and Hughes the source of bacteremia was related to CLABSI $[5,6]$. In our study secondary bacteremia was related to VAP in $72.7 \%$, to CLABSI in $18.2 \%$ and, UTI $9.1 \%$ respectively. The most common pathogen isolated was $\mathrm{P}$. 
aeruginosa, followed by $\mathrm{E}$. faecalis and $\mathrm{S}$. aureus.

Giacobbe et al. observed an increased risk for CLABSI in $25 \%$ of the patients with an ICU stay greater than 15 days, and up to $50 \%$ of risk in prolonged stay (30 days) [10]. In the study by Cataldo et al. they observed an incidence of CLABSI in $49 \%$ of their population in the ICU and had a mean of ICU stay of 13 days prior the isolation [11]. In our study we reported the positivity of cultures in the first 14 days of ICU stay and an increased amount of positivity in blood samples, increasing the percentage of secondary bacteremia, but still a lower percentage in CLABSI compared to Cataldo study.

The presence of a primary bacteremia was reported in $35.3 \%$ of the patients where Chryseobacterium indologenes was the most common pathogen isolated, followed by $\mathrm{E}$. coli, similar microorganisms' isolations were observed in other studies $[12,13]$. Also, we observed an increased requirement of vasopressor during their stay on the ICU, with a tendency of increase ICU stay without changes in hospital length of stay or mortality (OR of 0.87 ) with no statistical significance $(p=0.75)$; Similar to our findings Cataldo et al that did not reported changes in mortality with an overall ICU mortality of $32 \%$ [11]. However, it differs from Hughes that observed a relative risk for mortality of 1.5 on these patients [5].

Our study has several limitations. First, this is a single-center retrospective analysis with a relatively small sample size. Second, the follow-on time of 14 days, may generate an infra-estimation of positive cultures on patients with a prolonged length of stay. Third, several of the patients was already treated with antibiotics, prior to the ICU or hospital admission.

\section{Conclusions}

Critically ill patients with active SARS-CoV-2 infection and a bacteremia tend to increase the ICU length of stay, days on IMV and dosage of norepinephrine, which could end up in multiple hospital complication, such as, use of renal replacement therapy, ventilator-induced lung injury, requirement of a tracheostomy and other nosocomial infection. Nevertheless, the mortality has no change compared to patients without a bacteremia.

\section{Disclosure Statement}

The authors declare that they have no competing interests.

\section{Funding}

No financial support was provided in the development of this work.

\section{Acknowledgements}

We would like to acknowledge the efforts and work of all the first responders from Medica Sur in this COVID-19 pandemic and the microbiologic department of the central laboratory. This work would not be possible without all of you.

\section{References}

[1] World Health Organization. Weekly Epidemiological Update on COVID-19. 2020; (October). https://www.who.int/docs/default-source/coronaviruse/situatio n-reports/20201012-weekly-epi-update-9.pdf.

[2] Sosa-García JO, Gutiérrez-Villaseñor AO, García-Briones A, Romero-González JP, Juárez-Hernández E, González-Chon O. Experience in the management of severe COVID-19 patients in an intensive care unit. Cir y Cir (English Ed. 2020; 88 (5): 569-575. doi: 10.24875/CIRU.20000675.

[3] Colchero MA, Fuentes ML, Salinas CAA. La Obesidad En México.; 2018.

[4] Zhang $\mathrm{H}$, Zhang $\mathrm{Y}$, Wu J, et al. Risks and features of secondary infections in severe and critical III COVID-19 patients. Emerg Microbes Infect. 2020; 9 (1): 1958-1964. doi: $10.1080 / 22221751.2020 .1812437$.

[5] Hughes S, Troise O, Donaldson H, Mughal N, Moore LSP. Since January 2020 Elsevier has created a COVID-19 resource centre with free information in English and Mandarin on the novel coronavirus COVID- 19. The COVID-19 resource centre is hosted on Elsevier Connect, the company's public news and information. 2020; (January).

[6] Engsbro AL, Israelsen SB, Pedersen M, et al. Predominance of hospital-acquired bloodstream infection in patients with COVID-19 pneumonia. Infect Dis (Auckl). 2020; 52 (12): 919-922. doi: 10.1080/23744235.2020.1802062.

[7] Martinez-guerra BA, Gonzalez-lara MF, Nereyda A, et al. Antimicrobial resistance patterns and antibiotic use during hospital conversion in the COVID-19 pandemic FGL_BMG 30NOV. 2021: 1-9.

[8] Yu D, Ininbergs K, Hedman K, Giske CG, Strålin K, Özenci V. Low prevalence of bloodstream infection and high blood culture contamination rates in patients with COVID-19. PLoS One. 2020; 15 (11): e0242533. doi: 10.1371/journal.pone.0242533.

[9] Sligl WI, Dragan T, Smith SW. Nosocomial Gram-Negative Bacteremia in Intensive Care: Epidemiology, Antimicrobial Susceptibilities, and Outcomes. Vol 37. International Society for Infectious Diseases; 2015. doi: 10.1016/j.ijid.2015.06.024.

[10] Giacobbe DR, Battaglini D, Ball L, et al. Bloodstream infections in critically III patients with COVID-19. Eur J Clin Invest. 2020; 50 (10): 1-8. doi: 10.1111/eci.13319.

[11] Cataldo MA, Tetaj N, Selleri M, et al. Incidence of bacterial and fungal bloodstream infections in COVID-19 patients in intensive care: An alarming "collateral effect." J Glob Antimicrob Resist 23. 2020; 23: 290-291.

[12] Nori P, Cowman $\mathrm{K}$, Chen $\mathrm{V}$, et al. Bacterial and fungal co-infections in COVID-19 patients hospitalized during the New York city pandemic surge. Infect Control Hosp Epidemiol. 2020; 2020: 1-5. doi: 10.1017/ice.2020.368.

[13] Sepulveda J, Westblade LF, Whittier S, et al. Bacteremia and blood culture utilization during COVID-19 surge in New York City. J Clin Microbiol. 2020; 58 (8): 1-7. doi: 10.1128/JCM.00875-20. 